\title{
Rapid eye movement production: Effects of activation phases and traffic noises
}

\author{
LAURA D. WEBER and ALAIN MUZET \\ Centre d'Études Bioclimatiques du C.N.R.S., 21 rue Becquerel, 67087 Strasbourg Cedex, France
}

\begin{abstract}
The purpose of this study was to determine whether the occurrence of activation phases during REM sleep modified rapid eye movement production in any way, and, if so, whether this modification was in the form of an increase or a decrease in the rapid eye movement density. Eighteen subjects, ranging from 6 to 66 years in age, were studied during 4 consecutive nights in the laboratory. The first 2 nights were undisturbed baseline nights, while the last 2 were disturbed by traffic noises. Results showed that activation phases were not preceded by decreases or increases in the rapid eye movement density, activation phases interrupted and significantly delayed the following rapid eye movement production, and traffic noises of a moderate peak intensity (65 dBA) did not have an effect on rapid eye movement production.
\end{abstract}

Although there is a question as to whether or not all the constituents of REM sleep are perfectly known, there is one constituent that has been a subject of interest for several years now: rapid eye movements (REMs). First discovered by Aserinsky and Kleitman (1953), rapid eye movements are unique to REM sleep. This team and others, such as Aserinsky (1971) and Benoit, Parot, and Garma (1974), have been interested in various characteristics of REMs such as density. It was during the course of a recent study concerning rapid eye movement density that we became concerned with the presence of activation phases (phase d'activation transitoire, or PAT; see Schieber, Muzet, \& Ferriere, 1971) during REM sleep and their potential effects on the production of REMs. Thus, one purpose of this study was to determine whether the occurrence of PATs during REM sleep modified rapid eye movement production in any way, and, if so, whether this modification was in the form of an increase or a decrease in the rapid eye movement density.

In addition, we will examine the effects of an external stimulus, noise, on REMs. Although there exists an abundant amount of literature concerning the effects of noise on sleep (Griefahn, 1980; Muzet \& Naitoh, 1977; Thiessen, 1978), there is little information concerning their effects on REMs.

\section{METHOD}

The results from this study were derived from an experiment concerning the effects of noise on different age groups. This experiment consisted of three different phases, with the following common points: the nocturnal ambient conditions, which were

This research was supported by Grant 76-22 of the Ministère Français de l'Environnement et du Cadre de Vie. The authors would like to thank H. Jouannic for assisting with the statistical analysis. maintained at constant levels during all experimental nights (an air temperature of $20^{\circ} \pm 1^{\circ} \mathrm{C}$ and a relative humidity of $60 \% \pm 5 \%$ ); a background noise of $35 \mathrm{dBA}$ due to air conditioning; and six traffic noises varying from 40 to $65 \mathrm{dBA}$ in intensity and from 2 to $12 \mathrm{sec}$ in duration. During the disturbed nights of this study, experimental noises were diffused according to a semirandom temporal distribution at a rate of 90 noises $/ \mathrm{h}$.

In addition, upon retiring, each subject remained in bed until $0700 \mathrm{~h}$ the next morning in order to obtain the following continuous electrophysiological registrations: electroencephalogram derivations F3-A2 and C3-A3; two electrooculograms (the electrodes were placed slightly below and above the outer canthus); electromyogram of either the mentalis or the masseter; electrocardiogram; finger pulse amplitude; pulse wave velocity; and body mobility that was measured by a bed actograph and a radar detection system. An example of the electrophysiological registrations and of a PAT can be seen in Figure 1.

Each morning, immediately after their awakening, the subjects answered a questionnaire concerning their sleep. The questionnaire was adapted to the three different age groups used in this study.

All sleep records were visually scored for sleep stages by $30-\mathrm{sec}$ epochs, according to the criteria given by Rechtschaffen and Kales (1968). During REM phases, REMs were detected visually and counted by periods of $1 \mathrm{~min}$. Only REMs visible on both electrooculograms and having an amplitude of at least $5 \mathrm{~mm}$ $(60 \mu \mathrm{V})$ on one of the two channels were taken into consideration for this study. These 1 -min periods were used to determine overall rapid eye movement densities for the three age groups. Rapid eye movement density is defined here as the total number of REMs divided by the total number of minutes spent in REM sleep.

All PATs occurring during the reference nights that were preceded and followed by at least 2 min free of another PAT were used to determine their effects on REMs. We used PATs that were preceded and followed by 2 min free of other PATs because an increase in this 2-min limitation would have drastically decreased the number of cases that we could have used. A PAT is defined as a cortical activation seen on the EEG that may be coupled with one or several of the following modifications: a body movement; an increase in muscle tone; an increase in heart rate; and a finger vasoconstriction. In this instance, REMs were counted by 10-sec periods in the minute preceding and following a PAT. None of these PATs were associated with stage changes. After the occurrence of a PAT, both the EMG and the EEG were those typical of REM sleep, as can be seen in Figure 1.

In addition, a fictitious PAT (PATF) was situated in the middle of any 4-min period of REM without a PAT. Once again, REMs 


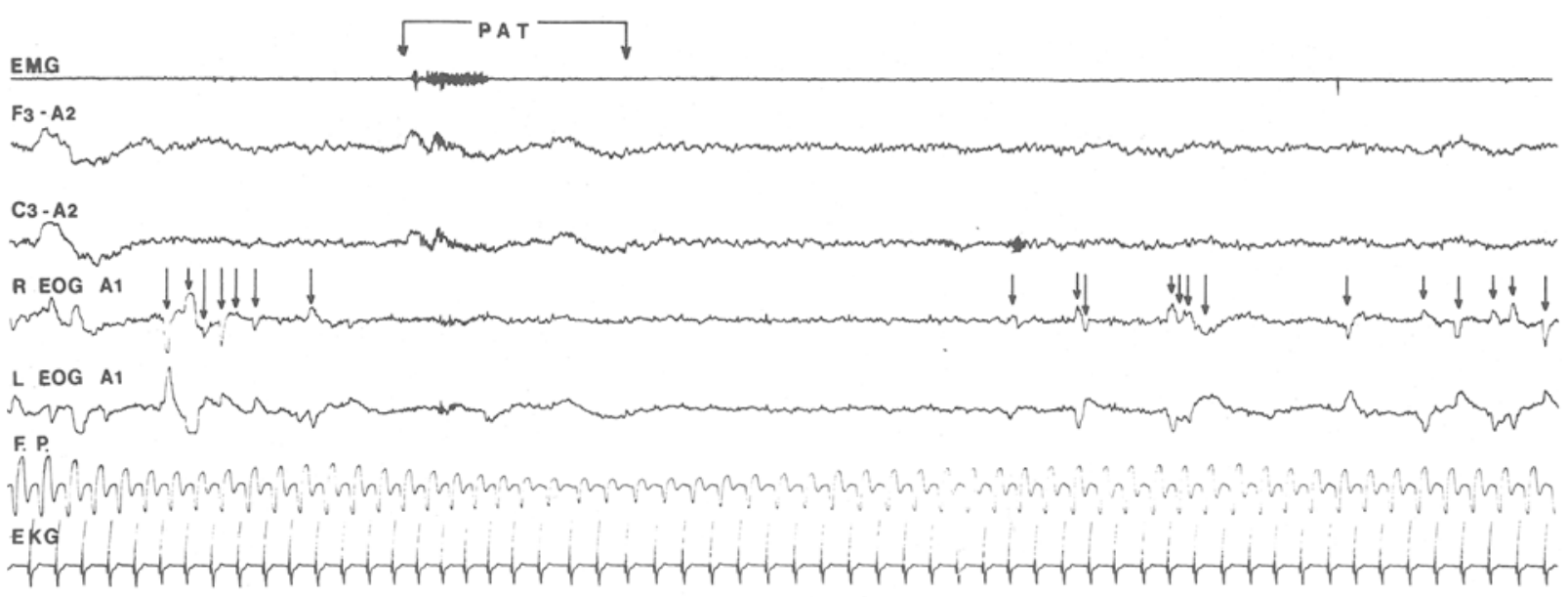

Acto bed

Radar

IiII

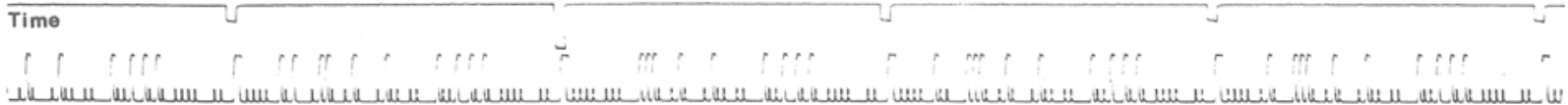

Figure 1. Example of an electrophysiological recording. From top to bottom: EMG of either mentalis or masseter; EEG derivations F3 and C3, which were referenced to the opposite mastoid, A2; right EOG and left EOG, which were referenced to the left mastoid, A1; finger pulse; EKG; bed actograph; radar detection of small body movements; time in 10-sec intervals; and the time code. The arrows indicate REMs and duration of a PAT.

were counted by $10-\mathrm{sec}$ periods in the 1 minute preceding and following a PATF. Approximately $40 \%$ of the PATs used in this study were located in either the first or last quarter of the REM period. The remaining $60 \%$ of the PATs occurred during the middle two quarters of the REM period. This proportion was also the same for the PATFs.

The individual aspects concerning the three phases used in this study were the following. In Phase 1 , six young adults between 19 and 24 years of age (three of each sex) were recorded. These subjects spent 20 consecutive nights in the laboratory. However, only Nights 1 through 4 were used in this study. Night 1 (N1) and Night 2 (N2) were baseline nights, while Night 3 (N3) and Night 4 (N4) were disturbed by traffic noises. "Lights out" was at $2300 \mathrm{~h}$, and sleep polygraph registrations in addition to noise diffusion during the disturbed nights were put into effect, without interruption, from $2300 \mathrm{~h}$ until $0700 \mathrm{~h}$ the next morning. In Phase 2, six children between 6 and 12 years of age (three of each sex) were registered in the laboratory during 4 consecutive nights. N1 and $\mathrm{N} 2$ were baseline nights, and N3 and N4 were disturbed by traffic noises. "Lights out" was at their usual bedtime hour, and sleep polygraph recordings were put into effect, without interruption, until $0700 \mathrm{~h}$ the next morning. During N3 and N4, noises were diffused only after the subject had fallen asleep. In the case of a nocturnal awakening, the noise was interrupted until the subject had once again fallen asleep. In Phase 3, six elderly people between 56 and 66 years of age (three of each sex) were recorded. The experimental conditions for this phase were identical to those of Phase 2.

An ANOVA was used in this study, and only results that were significant at the $5 \%$ level or better will be presented.

\section{RESULTS}

No significant within-groups differences were found between $\mathrm{N} 1$ and $\mathrm{N} 2$, either in the PAT fre- quency or in the rapid eye movement density during REM sleep. The same was found to be true for N3 and N4. Therefore, to facilitate analysis, N1 was combined with N2, and N3 was combined with N4. $\mathrm{N} 1$ and N2 were separated from N3 and N4 because experimental conditions were not the same, due to the presence or absence of traffic noises.

\section{Rapid Eye Movement Density Before and After PATs and PATFs}

A four-way ANOVA was used in order to determine whether or not there were any trends in the rapid eye movement density both before and after PATs and PATFs. This procedure exposed a significant trend only in the rapid eye movement density after PATs $[F(5,102)=3.6, p<.005]$. In addition, a two-way ANOVA with repeated measures was performed in order to expose any differences between the rapid eye movement density before and after PATs and PATFs. Results showed significant differences for the first level of analysis [before vs. after: $F(11,408)=12.1, p<.001]$, the second level of analysis [PAT vs. PATF: $F(11,408)=3.68, p<.001$ ], and at the level of interaction $[F(11,408)=6.46$, $\mathrm{p}<.001$ ]. A priori tests were used to precisely locate significant differences between the rapid eye movement density before and after PATs and PATFs. Results showed that significant differences existed between the rapid eye movement density before and 
after PATs $[t(408)=3.3, p<.01]$ and the rapid eye movement density after PATs and PATFs $[t(408)=$ $3.1, p<.01]$. Represented in Figure 2 are the results from the analysis of the rapid eye movement density per 10 -sec epochs occurring $1 \mathrm{~min}$ before and after the PATs and PATFs. The solid line represents the mean of 155 PATs, and the broken line represents the mean of 251 PATFs. This figure shows that the rapid eye movement density both before and after the PATFs is very similar to the rapid eye movement density before the PATs. However, there is a significant change in the rapid eye movement density after the PAT, that change being in the form of a significant decrease. These results are in accordance with the results from the analysis of variance presented earlier.

\section{Rapid Eye Movement Latency \\ After PATs and PATFs}

The time between the end of a PAT and the beginning of the first rapid eye movement following the PAT is defined as being the rapid eye movement latency. The rapid eye movement latency was measured for each PAT as well as each PATF. The PATFs that were used for this part of the study were situated 2 min after the end of a PAT. The histogram represented in Figure 3 shows that only $6.7 \%$ of the PATs of the baseline nights were followed by the first rapid eye movement within the 10 -sec period occurring immediately after the end of the PAT. However, for the same baseline nights, $61 \%$ of the PATFs were followed by the first rapid eye movement within this 10 -sec period. These same percentages for the PATs and PATFs of the disturbed nights were $11.8 \%$ and $54 \%$, respectively. The calculation of the average interval occurring between the end of the PAT and the first rapid eye movement for the baseline nights was $42.5 \mathrm{sec}$, while for the PATFs of these same nights the average interval was $15.5 \mathrm{sec}$. The same difference was found to exist between the PATs and the PATFs occurring during the disturbed nights (PATs $=39.3 \mathrm{sec}$ and PATFs $=17.3 \mathrm{sec}$ ).

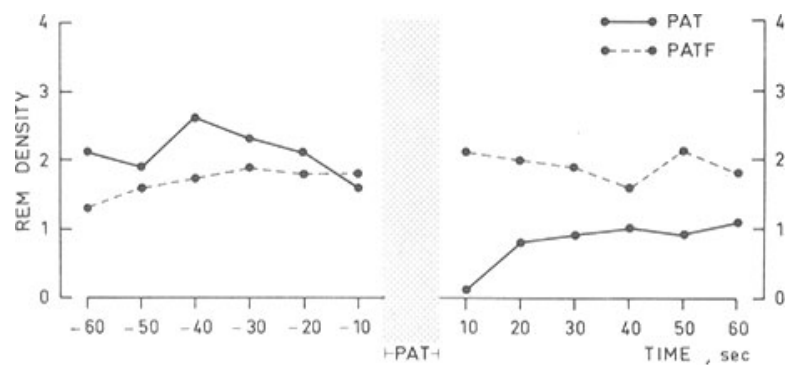

Figure 2. Evolution of average rapid eye movement density per 10-sec period, during the minute preceding and following PATs (solid line) and PATFs (broken line).

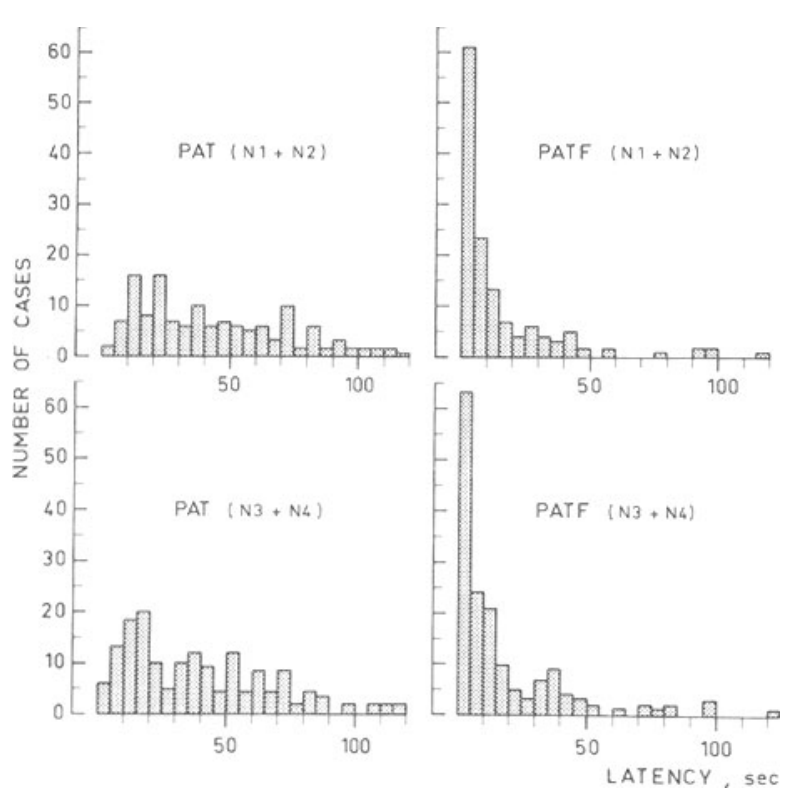

Figure 3. Distribution of the interval occurring between the beginning of the first rapid eye movement and the end of the preceding PAT or PATF during undisturbed nights (N1 and N2) and disturbed nights (N3 and N4). The mean interval following PATs during $\mathrm{N1}$ and $\mathrm{N2}$ was $\mathbf{4 2 . 5} \mathrm{sec}$, and for PATFs of these same nights was 15.5 sec. The figures for PATFs of N3 and N4 were 39.3 and $17.3 \mathrm{sec}$, respectively.

\section{The Effects of Noise on Rapid Eye Movements}

All noises diffused during N3 and N4 in the three phases of this study appeared on the polygraph records. This allowed us to keep track of the temporal location, the beginning, and the end of any given noise. Also, fictitious noises appeared in the same manner on all polygraph records of $\mathrm{N} 1$ and $\mathrm{N} 2$, although noises were not introduced into the sleep chamber. All noises with a peak intensity of $65 \mathrm{dBA}$ occurring during the disturbed nights that were both preceded and followed by at least $2 \mathrm{~min}$ free of PATs were examined to determine their effects on REMs. The distance between the end of a noise and the beginning of the first rapid eye movement is again defined as being the rapid eye movement latency. In addition, the rapid eye movement latency of all fictitious noises of the baseline nights that were preceded and followed by at least 2 min free of another PAT were equally measured. Again, the average interval occurring between the end of a noise and the beginning of the first eye movement was calculated and found to be $9.9 \mathrm{sec}$. The mean interval for the undisturbed baseline nights was $9.6 \mathrm{sec}$.

The results represented in Figure 4 show that $63 \%$ of the rapid eye movement latencies occurring during the disturbed nights were less than $10 \mathrm{sec}$. This percentage was nearly the same for the rapid eye movement latencies occurring during the baseline nights $(66 \%)$. 


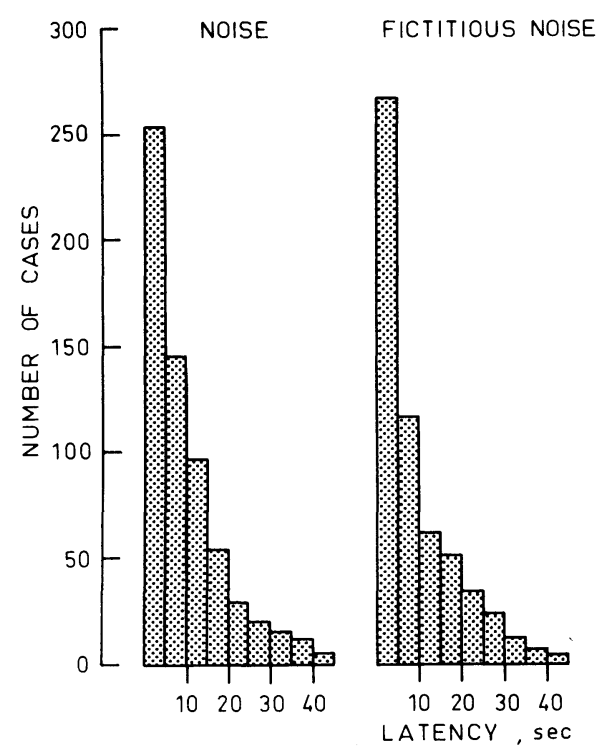

Figure 4. Distribution of the interval occurring between the beginning of the first rapid eye movement and the end of the preceding noise (65 dBA) and fictitious noise. The mean interval following the noise was $9.9 \mathrm{sec}$, and following the fictitious noise it was $9.6 \mathrm{sec}$.

\section{DISCUSSION}

The presence of PATs throughout the sleep cycle has been a point of interest for some time now. Although their function during sleep remains unclear, some headway has been made toward an explanation. It has been shown that the total number of PATs occurring during the night remains very stable from one night to the next in the same subjects, even under varying conditions (Ehrhart \& Muzet, 1974). They occur with different frequencies during different stages of sleep; and, during REM sleep, PATs are usually less intense and appear more frequently than in other sleep stages (Ehrhart \& Muzet, 1974). It is important to note that even though PATs are generally scored as "body movement artifact," with very sensitive recording of body mobility it can be shown that they are not always accompanied by body movements. However, PATs, whether associated with body movements or not, are preceded by an increase in the heart rate beginning approximately 7 to 8 beats before the onset of the PAT (Muzet \& Michel, 1977). Therefore, it is possible that motor activity associated with PATs occurring during REM sleep can be explained by a partial and temporary interruption of the descending motor inhibition. PATs have also been shown to be capable of modifying the sleep structure, in addition to being important predictors of stage change (Muzet, Naitoh, Townsend, \& Johnson, 1972).

The results of this study have shown that: (1) PATs occurring during REM sleep were not preceded by decreases or increases in rapid eye movement density in the $1 \mathrm{~min}$ prior to a PAT; (2) the occurrence of PATs was associated with both an interruption of rapid eye movement production and a significant delay in the occurrence of the following rapid eye movement; and (3) traffic noises of moderate peak intensity (65 dBA) did not have an effect on rapid eye movement production. Therefore, it can be said that rapid eye movement production, not having been affected by external stimuli (noise) was, on the contrary, affected by PATs.

In conclusion, these findings may have important implications for the understanding of PATs and their effects on certain REM sleep constituents such as rapid eye movement density and rhythmicity of rapid eye movement production.

\section{REFERENCES}

Aserinsky, E. Rapid eye movement density and pattern in the sleep of normal young adults. Psychophysiology, 1971, 8, 361-375.

ASERINSKy, E., \& Kleitman, N. Regularly occurring periods of eye mobility and concomitant phenomenon during sleep. Science, 1953, 118, 273-274.

Benoit, O., Parot, S., \& Garma, L. Evolution during the night of REM sleep in man. Electroencephalography and Clinical Neurophysiology, 1974, 36, 245-251.

Ehrhart, J., \& Muzet, A. Frequence et durée des phases d'activation transitoire au cours du sommeil normal ou perturbé chez l'homme. Extrait des Archives des Science Physiologique, 1974, $28,3$.

Griefahn, B. Research on noise-disturbed sleep since 1973. Noise as a public health problem: Proceedings of the Third International Congress, 1980, 10, 377-390.

Muzet, A., \& Michel, C. Heart rate preceding short activation phases in sleep. Waking and Sleeping, 1977, 1, 175-179.

Muzet, A., \& Naitoh, P. Sommeil et bruit. Extrait de Confrontation Psychiatriques, 1977, 15, 215-235.

Muzet, A., Naitoh, P., Townsend, R. E., \& Johnson, L. C. Body movements during sleep as a predictor of stage change. Psychonomic Science, 1972, 29, 7-10.

Rechtschaffen, A., \& Kales, A. (Eds.). A manual of standardized terminology, techniques and scoring systems for sleep stages of human subjects. Washington, D.C: U.S. Government Printing Office (Public Health Service), 1968.

Schieber, J. P., Muzet, A., \& Ferriere, P. J. Les phases d'activation transitoire spontanées au cours du sommeil normal chez l'homme. Archives des Sciences Physiologiques, 1971, 25, 443-465.

Thiessen, G. J. Disturbance of sleep by noise. Journal of the Acoustical Society of America, 1978, 64, 216-222.

(Manuscript received March 2, 1981; revision accepted for publication June 2, 1981.) 